\title{
CHARACTERIZATION OF OPTIMAL STOPPING REGIONS OF AMERICAN ASIAN AND LOOKBACK OPTIONS
}

\author{
Min Dai \\ Department of Mathematics, National University of Singapore, Singapore \\ Yue Kuen Kwok \\ Department of Mathematics \\ Hong Kong University of Science and Technology \\ Clear Water Bay, Hong Kong, China
}

\begin{abstract}
A general framework is developed to analyze the optimal stopping (exercise) regions of American path dependent options with either Asian feature or lookback feature. We examine the monotonicity properties of the option values and stopping regions with respect to the interest rate, dividend yield and time. From the ordering properties of the values of American lookback options and American Asian options, we deduce the corresponding nesting relations between the exercise regions of these American options. We illustrate how some properties of the exercise regions of the American Asian options can be inferred from those of the American lookback options.
\end{abstract}

Key Words: American options, optimal stopping, Asian feature, lookback feature, monotonicity properties

\section{INTRODUCTION}

The path dependence in an option payoff is signified by the dependence of the payoff function on the path history of the underlying asset price. The two most common path dependent features involve either taking some form of averaging or recording the extremum value of the asset price path. Options with averaging payoff are called Asian options while those options whose payoff depends on some extremum value are called lookback options. The American feature entitles the right to the option holders to exercise the option prematurely. These added contractual features help option holders capitalize their view on the future movement of the underlying asset price or hedge against certain type of risk exposure.

The price function of an American path dependent option is known to be governed by parabolic variational inequalities. Besides option value, the solution of the variational inequalities involves the determination of the time dependent free boundary at which optimal stopping occurs, that is, the holder should exercise the option optimally at some threshold asset price level. The domain of the option pricing model is divided into two regions by the optimal stopping boundary, namely, the continuation region where the holder should continue to hold the option and the stopping region (exercise region) where the option should be optimally exercised. In this paper, we develop a general framework using partial differential equation theory to analyze the monotonicity properties of the price functions and the optimal exercise policies of American path dependent options. In particular, we examine the characterization of the exercise regions of American options with different path dependent payoffs.

Address correspondence to Yue Kuen Kwok, Department of Mathematics, Hong Kong University of Science and Technology, Clear Water Bay, Hong Kong; email: maykwok@ust.hk. 
There have been numerous papers on the valuation of American Asian options (Wu et al., 1999; Hansen and Jorgensen, 2000; Ben-Ameur et al., 2002; Marcozzi, 2003; Wu and Fu, 2003) and American lookback options (Dai, 2001; Yu et al., 2001; Lai and Lim, 2004; Dai and Kwok, 2005) under the Black-Scholes framework. Most of these papers deal with the construction of algorithms for numerical valuation of the values and exercise boundaries of the American path dependent option models. The numerical approaches range from the enhanced lattice tree methods, simulation based algorithms, iterative solution of integral equation, variational approach and Bernoulli random walk approximation. The valuation of path dependent options involves typically non-Markovian systems in terms of the asset price process. The partial differential equation formulation involves an additional path dependent state variable. A collection of interesting results on the analytic properties of the values and exercise boundaries have been reported. For example, Hansen and Jorgensen (2000) observe the non-monotone property of the exercise boundaries of American Asian call and put options with respect to the calendar time. Wu and Fu (2003) prove the convexity property of the exercise boundary under certain assumption of the asset price process.

While most of the above papers consider the pricing of American Asian options under the Black-Scholes framework, there have been some recent interests to consider pricing of American Asian options under more general asset price processes. The dimension of the pricing model increases by one by the introduction of stochastic volatility as an additional state variable. Also, solution to the option value usually can only be represented analytically in the Fourier space. Under the jump-diffusion assumption, the pricing formulation becomes a parabolic integro-differential free boundary value problem (Pham, 1997). The early exercise premium contains an extra term due to jumps, which is related to the adjustment cost made by the option holder when the underlying asset price jumps from the stopping region back into the continuation region (Gukhal, 2001; Chiarella and Ziogas, 2004). Parott and Clarke (1998) consider the parallel computation of American Asian options with stochastic volatility. Also, d' Halluin et al. (2003) uses the semi-Lagrangian approach to solve the governing partial integro-differential equation when the asset price follows the jumpdiffusion process. The theoretical studies of the pricing properties of American Asian options under the stochastic volatility process or jump-diffusion process remain unsolved challenging mathematical problems.

As the averaging payoff structure looks very differently from the lookback payoff, it appears that there is little resemblance between the exercise policies of American lookback options and American Asian options. The objective of our work is to provide a general framework of deriving analytic properties of the option value and exercise region of an American option with either averaging or lookback payoff. We show how some analytic properties of the exercise regions of the American Asian options can be inferred from those of the American lookback counterparts. This paper is organized as follows. In the next section, we present the setup of our pricing models and notations. We then consider the existence properties of the exercise regions of American path dependent options and the nesting relations between the option values and exercise regions. Some analytic properties of the optimal stopping boundaries of the American floating strike and fixed strike Asian options are derived. In Section 3, we provide theoretical proofs for the monotonicity properties of the option values and exercise boundaries of American Asian and lookback options with respect to the calendar time, interest rate and dividend yield. The last section presents our conclusive remarks on the characterization of optimal stopping policies of American path dependent options.

\section{EXERCISE REGIONS OF AMERICAN PATH DEPENDENT OPTIONS}

We consider the usual Black Scholes economy with a risky asset and a money market account. We assume the existence of a risk neutral probability $Q$ under which discounted asset prices are martingales, implying 
non-existence of arbitrage. Under the pricing measure $Q$, the asset price is assumed to follow

$$
\frac{d S_{t}}{S_{t}}=(r-q) d t+\sigma d Z_{t},
$$

where $r$ and $q$ are the constant riskless interest rate and dividend yield, respectively, $\sigma$ is the constant instantaneous volatility of asset return and $Z$ is a $Q$-Brownian motion. Let $t$ and $T$ denote the current time and date of expiration of the option, respectively. The path dependent variable $J_{t}$ may take some form of averaging or record an extremum value of the asset price path over the time period $[0, t]$. We assume that the option's path dependent payoff structure depends on $J_{t}$ and possibly $S_{t}$ also. Let $\Lambda\left(S_{t}, J_{t} ; K\right)$ denote the payoff function of an American path dependent option. The common forms of $\Lambda\left(S_{t}, J_{t} ; K\right)$ are

$$
\Lambda\left(S_{t}, J_{t} ; K\right)= \begin{cases}\left(S_{t}-J_{t}\right)^{+} & \text {floating call } \\ \left(J_{t}-S_{t}\right)^{+} & \text {floating put } \\ \left(J_{t}-K\right)^{+} & \text {fixed call } \\ \left(K-J_{t}\right)^{+} & \text {fixed put }\end{cases}
$$

where $K$ is the fixed strike and

$$
x^{+}=\left\{\begin{array}{ll}
x & \text { if } x \geq 0 \\
0 & \text { if } x<0
\end{array} .\right.
$$

Suppose continuous monitoring of the asset price path is assumed, we define

(i) arithmetic averaging $A_{t}$

$$
A_{t}=\frac{1}{t} \int_{0}^{t} S_{u} d u
$$

(ii) minimum value $m_{t}$ and maximum value $M_{t}$

$$
m_{t}=\min _{u \in[0, t]} S_{u} \quad \text { and } \quad M_{t}=\max _{u \in[0, t]} S_{u} .
$$

By choosing $J_{t}$ to be either $A_{t}, m_{t}$ or $M_{t}$, a wide variety of path dependent option payoffs can be structured. Some examples are

(i) floating strike lookback call

$$
\Lambda\left(S_{t}, J_{t} ; K\right)=\left(S_{t}-J_{t}\right)^{+}, \text {where } J_{t}=m_{t} ;
$$

(ii) fixed strike lookback put

$$
\Lambda\left(S_{t}, J_{t} ; K\right)=\left(K-J_{t}\right)^{+} \text {, where } J_{t}=m_{t} \text {; }
$$

(iii) floating strike Asian put

$$
\Lambda\left(S_{t}, J_{t} ; K\right)=\left(J_{t}-S_{t}\right)^{+}, \text {where } J_{t}=A_{t} ;
$$

(iv) fixed strike Asian call

$$
\Lambda\left(S_{t}, J_{t} ; K\right)=\left(J_{t}-K\right)^{+} \text {, where } J_{t}=A_{t} .
$$


Consider an American path dependent option with payoff function $\Lambda\left(S_{t}, J_{t} ; K\right)$, its no-arbitrage price is given by (Jaillet et al., 1990)

$$
V(S, J, t)=\sup _{t^{*} \in \mathcal{T}_{t, T}} E_{Q}\left[e^{-r\left(t^{*}-t\right)} \Lambda\left(S_{t^{*}}, J_{t^{*}} ; K\right) \mid S_{t}=S, J_{t}=J\right]
$$

where $E_{Q}$ denotes the expectation under the risk neutral measure $Q, t^{*}$ is the optimal stopping time and $\mathcal{T}_{t, T}$ is the set of stopping times taking values between $t$ and $T$. The price function $V(S, J, t)$ is defined in the domain $D \times[0, T]$, where $D$ takes the form

$$
D= \begin{cases}\{0<S<\infty, 0<J<\infty\} & \text { for Asian option } \\ \{0<S \leq J<\infty\} & \text { for lookback maximum option } \\ \{0<J \leq S<\infty\} & \text { for lookback minimum option }\end{cases}
$$

The variational inequalities that govern $V(S, J, t)$ are given by (Jaillet et al., 1990)

$$
\begin{aligned}
& \frac{\partial V}{\partial t}+\mathcal{L} V \geq 0 \quad \text { in } D \times(0, T), \quad V \geq \Lambda \quad \text { on } \bar{D} \times[0, T), \\
& \left(\frac{\partial V}{\partial t}+\mathcal{L} V\right)(V-\Lambda)=0 \quad \text { in } D \times(0, T),
\end{aligned}
$$

with terminal payoff

$$
V(S, J, T)=\Lambda(S, J) \text { in } D .
$$

The differential operator $\mathcal{L}$ takes the form

$$
\mathcal{L}=\left\{\begin{array}{ll}
\frac{\sigma^{2}}{2} S^{2} \frac{\partial^{2}}{\partial S^{2}}+(r-q) S \frac{\partial}{\partial S}+\frac{S-J}{t} \frac{\partial}{\partial J}-r & \text { for Asian option } \\
\frac{\sigma^{2}}{2} S^{2} \frac{\partial^{2}}{\partial S^{2}}+(r-q) S \frac{\partial}{\partial S}-r & \text { for lookback option }
\end{array} .\right.
$$

In the exercise region $E$, the price function equals the payoff so that

$$
E=\{(S, J, t) \in D \times[0, T): \quad V(S, J, t)=\Lambda(S, J)\} .
$$

To characterize the exercise region more precisely, we define $E^{t}$ to be the exercise region at time $t$, where

$$
E^{t}=\{(S, J) \in D: \quad V(S, J, t)=\Lambda(S, J)\}, \quad t \in[0, T)
$$

\section{Remarks}

1. Since the differential operator $\mathcal{L}$ in the Asian option model contains the factor $\frac{S-J}{t}$, the finite-lived Asian option price function has dependence on the calendar time $t$ and time to expiry $\tau, \tau=T-t$. For the perpetual case, $T \rightarrow \infty$, the perpetual Asian option price function still retains the dependence on the calendar time.

2. For lookback option models, we have to append the following boundary condition in the variational inequalities formulation

$$
\left.\frac{\partial V}{\partial J}\right|_{S=J}=0
$$


The above normal reflection condition arises from the observation that the option value is insensitive to the current extremum value $J$ when the current asset value equals the current extremum value.

3. Even when the exercise region $E$ is non-empty, $E^{t}$ can be empty for some value of $t$. For notational convenience, we denote the exercise region at time $t$ of an American floating strike lookback call and an American fixed strike Asian put by $E_{L, f c}^{t}$ and $E_{A, x p}^{t}$, respectively, and similar notations for other types of American path dependent options.

For finite-lived American lookback options, the characterization of the exercise region $E_{L}^{t}$ has been analyzed in our earlier paper (Dai and Kwok, 2005). The main results on the existence of $E_{L}^{t}$ are summarized in Lemma 1.

\section{Lemma 1}

Let $E_{L}^{t}$ denote the exercise region of a finite-lived American lookback option.

(i) When $q \neq 0, E_{L}^{t} \neq \emptyset$.

(ii) When $q=0, E_{L}^{t}=\emptyset$ for the American floating strike lookback call but $E_{L}^{t} \neq \emptyset$ for all other types of American lookback options.

The financial intuition behind the forfeiture of the early exercise right in an American floating strike lookback call when $q=0$ is quite obvious. It is well known that it is never optimal to exercise an usual American call option when the underlying asset is non-dividend paying. The American floating strike lookback call has the additional privilege of resetting the strike to the newly realized minimum value of the asset price, so $E_{L, f_{c}}^{t}=\emptyset$ when $q=0$.

\section{Ordering properties of price functions}

The price functions of the American lookback option and its Asian option counterpart can be deduced to satisfy some ordering properties. Let $V_{L, f c}(S, J, t)\left[V_{A, f c}(S, J, t)\right]$ denote the price function of the American floating strike lookback (Asian) call option, where

$$
\begin{aligned}
& V_{L, f c}(S, J, t)=\sup _{t^{*} \in \mathcal{T}_{t, T}} E_{Q}\left[e^{-r\left(t^{*}-t\right)}\left(S-m_{t^{*}}\right) \mid m_{t}=J, S_{t}=S\right] \\
& V_{A, f c}(S, J, t)=\sup _{t^{*} \in \mathcal{T}_{t, T}} E_{Q}\left[e^{-r\left(t^{*}-t\right)}\left(S-A_{t^{*}}\right)^{+} \mid A_{t}=J, S_{t}=S\right] .
\end{aligned}
$$

Jiang and Dai (2004) derive the following ordering property on the price functions

$$
V_{A, f c}(S, J, t) \leq V_{L, f c}(S, J, t), \quad \text { for } \quad S \geq J .
$$

Actually, we can deduce in a similar manner that the value of an American Asian option is always less than or equal to that of the lookback counterpart with the same values of $S, J$ and $t$.

\section{Nesting relations of the exercise regions}

Based on the above ordering properties of the price functions of the American lookback options and their Asian option counterparts, we can deduce the corresponding nesting relations of the exercise regions of these options. The results are summarized in Lemma 2.

\section{Lemma 2}


Let $E_{A}^{t}$ denote the exercise region of an American Asian option and $E_{L}^{t}$ denote the exercise region of the American lookback option counterpart. We always have

$$
E_{A}^{t} \subseteq E_{L}^{t}
$$

independent on whether it is a call or put, floating or fixed type.

The validity of the nesting relations in Lemma 2 can be shown by financial argument as follows. For a given set of values of $S_{t}$ and $J_{t}$ at time $t$, suppose it is optimal to exercise an American floating strike lookback call, then it must be optimal to exercise its Asian option counterpart. This is because the exercise payoffs are the same for both options, and the Asian call has a lower value if it stays unexercised. Such policy of continued holding of the Asian call is non-optimal.

\section{Existence of exercise regions}

From the results in Lemmas 1 and 2 , we can deduce that $E_{A}^{t} \neq \emptyset$ when $q \neq 0$. Also, when $q=0, E_{A}^{t} \neq \emptyset$ for American Asian options other than the floating strike call. We shall show by theoretical argument that the exercise boundary of an American floating strike Asian call option exists at least for times close to expiry, so we can claim that $E_{A, f c} \neq \emptyset$ though it is not sufficient to claim conclusively that $E_{A, f c}^{t} \neq \emptyset$ for all $t \in[0, T)$. We performed extensive numerical experiments to search for the possibility of $E_{A, f c}^{t}$ being empty. So far, our calculations have always revealed the non-emptiness of $E_{A, f c}^{t}$.

\section{Optimal stopping boundaries of floating strike Asian options}

For Asian options with floating strike payoff structure, we can achieve dimension reduction of the pricing model by defining the following similarity variables:

$$
x=A / S \quad \text { and } \quad W(x, \tau)=V(S, A, t) / S \quad \text { where } \quad \tau=T-t
$$

The variational inequalities for an American floating strike Asian option can be reformulated as

$$
\begin{aligned}
& \frac{\partial W}{\partial \tau}-\frac{\sigma^{2}}{2} x^{2} \frac{\partial^{2} W}{\partial x^{2}}+\left[(r-q) x-\frac{1-x}{T-\tau}\right] \frac{\partial W}{\partial x}+q W \geq 0 \quad \text { in } \quad(0, \infty) \times(0, T) \\
& W \geq \eta(1-x) \text { in }[0, \infty) \times[0, T) \\
& \left\{\frac{\partial W}{\partial \tau}-\frac{\sigma^{2}}{2} x^{2} \frac{\partial^{2} W}{\partial x^{2}}+\left[(r-q) x-\frac{1-x}{T-\tau}\right] \frac{\partial W}{\partial x}+q W\right\}[W-\eta(1-x)]=0 \\
& \text { in }(0, \infty) \times(0, T)
\end{aligned}
$$

with auxiliary condition: $W(x, 0)=[\eta(1-x)]^{+}$. Here, $\eta=1$ for the call and $\eta=-1$ for the put. The $x$-derivative of the obstacle function $\eta(1-x)$ in the above parabolic variational inequalities is negative (positive) for the call (put). By applying the comparison principle, we deduce that

$$
\begin{aligned}
& \text { (i) } \frac{\partial W}{\partial x} \leq 0 \text { for an American floating strike Asian call, } \\
& \text { (ii) } \frac{\partial W}{\partial x} \geq 0 \text { for an American floating strike Asian put. }
\end{aligned}
$$

Let $x^{*}(\tau)$ denote the optimal stopping boundary associated with the parabolic variational inequalities (2.7). We would like to show that the optimal stopping region of an American floating strike call (put) lies 
on the left (right) hand side of the optimal stopping boundary. Also, we examine the behavior of $x^{*}(\tau)$ as $\tau \rightarrow 0^{+}$. The results are summarized in the following lemmas.

\section{Lemma 3}

For the American floating strike Asian call, the optimal stopping region is

$$
E_{A, f_{c}}=\left\{(x, \tau): x \leq x^{*}(\tau), \tau>0\right\}
$$

while that of the put option counterpart is

$$
E_{A, f p}=\left\{(x, \tau): x \geq x^{*}(\tau), \tau>0\right\} .
$$

\section{Remark}

The American floating strike lookback call and put options possess similar properties of the optimal stopping regions (see Dai and Kwok, 2005).

\section{Proposition 4}

Let $x^{*}\left(0^{+}\right)$denote the value of the optimal stopping boundary at time close to expiry. We have

(i) floating strike Asian call

$$
x^{*}\left(0^{+}\right)=\min \left(\frac{1+q T}{1+r T}, 1\right)
$$

(ii) floating strike Asian put

$$
x^{*}\left(0^{+}\right)=\max \left(\frac{1+q T}{1+r T}, 1\right) .
$$

\section{Remark}

In terms of the similarity variable $x=J / S$, where $J$ can be the realized maximum value $M$ or realized minimum value $m$, the asymptotic behavior of the optimal stopping boundary at time close to expiry for the American floating strike lookback call and put are $x^{*}\left(0^{+}\right)=\min \left(\frac{q}{r}, 1\right)$ and $x^{*}\left(0^{+}\right)=\max \left(\frac{q}{r}, 1\right)$, respectively (see Dai and Kwok, 2005).

The proof of Lemmas 3 and 4 are presented in the Appendix.

\section{Optimal stopping boundaries of fixed strike Asian options}

Consider the American fixed strike Asian call option whose payoff is $(A-K)^{+}$. In the exercise region, the option value $V$ satisfies $V=A-K$ and

$$
\frac{\partial V}{\partial \tau}-\frac{S-A}{T-\tau} \frac{\partial V}{\partial A}-\frac{\sigma^{2}}{2} S^{2} \frac{\partial^{2} V}{\partial S^{2}}-(r-q) S \frac{\partial V}{\partial S}+r V \geq 0
$$


In addition, we have $\frac{\partial V}{\partial S} \geq 0$. Substituting $V=A-K$ into the above inequality, we obtain

$$
A-S+r T A-r T K \geq 0 \quad \text { or } \quad A>\frac{S+r T K}{1+r T} .
$$

This would imply that any point $(S, A, t)$ inside the exercise region must observe the above inequality. Now, for a given value of $\tau$, suppose $V\left(S_{1}, A, \tau\right)>A-K$, then $V\left(S_{2}, A, \tau\right)>A-K$ for $S_{2}>S_{1}$. The surface of the exercise region can then be parameterized by $A^{*}(S, \tau)$, provided that the exercise region exists. Also, we can derive some interesting properties on $A^{*}\left(S, 0^{+}\right), A^{*}\left(0^{+}, \tau\right)$ and $\lim _{S \rightarrow \infty} \frac{A^{*}(S, \tau ; K)}{S}$. For the American fixed strike Asian call option, these properties are summarized in Proposition 5.

\section{Proposition 5}

Let $A^{*}(S, \tau)$ represent the exercise boundary surface of an American fixed strike Asian call option. The asymptotic properties of $A^{*}(S, \tau)$ at $\tau \rightarrow 0^{+}, S \rightarrow 0^{+}$and $S \rightarrow \infty$ are given by

$$
A^{*}\left(S, 0^{+}\right)=\max \left(K, \frac{S+r T K}{1+r T}\right), \quad A^{*}\left(0^{+}, \tau\right)=K,
$$

and

$$
\lim _{S \rightarrow \infty} \frac{A^{*}(S, \tau ; K)}{S}=\frac{A^{*}(S, \tau ; 0)}{S}=\xi(\tau)
$$

where $\xi(\tau)$ is some function of $\tau$.

\section{Remarks}

1. The exercise boundary surface $M^{*}(S, \tau)$ of the American fixed strike lookback call option with payoff function $(M-K)^{+}$possesses similar asymptotic behaviors at $\tau \rightarrow 0^{+}, S \rightarrow 0^{+}$and $S \rightarrow \infty$ (see Dai and Kwok, 2005), where

$$
M^{*}\left(S, 0^{+}\right)=K, \quad M^{*}\left(0^{+}, \tau\right)=K,
$$

and

$$
\lim _{S \rightarrow \infty} \frac{M^{*}(S, \tau ; K)}{S}=\frac{M^{*}(S, \tau ; 0)}{S} .
$$

2. Similarly, we can deduce that the exercise boundary surface $A^{*}(S, \tau)$ of an American fixed strike Asian put satisfies

$$
A^{*}\left(S, 0^{+}\right)=\min \left(K, \frac{S+r T K}{1+r T}\right) \quad \text { and } \quad A^{*}(\infty, \tau)=K .
$$

3. The exercise boundary surface $m^{*}(S, \tau)$ of the American fixed strike lookback put option with payoff function $(K-m)^{+}$observes

$$
m^{*}\left(S, 0^{+}\right)=K \quad \text { and } \quad m^{*}(\infty, \tau)=K .
$$


The proof of Proposition 5 is presented in the Appendix.

\section{MONOTONICITY PROPERTIES WITH RESPECT TO DIVIDEND YIELD, INTEREST RATE AND TIME}

First, we would like to recall some well known results on the monotonicity properties of American vanilla option values with respect to the dividend yield $q$ and riskless interest rate $r$. Since the dividend yield tends to reduce the risk neutralized drift rate of the underlying asset price, it is intuitively clear that the value of vanilla call (put) decreases (increases) with increasing $q$. However, the impact of interest rate $r$ on the option value is slightly more complicated since $r$ appears both in the discount factor and the risk neutralized drift rate. For vanilla put options, an increase in $r$ would decrease both the discount factor and expected payoff so that the put value decreases with an increase in $r$. However, the effect of $r$ on the discount factor and expected payoff are counteracting in call options. Therefore, it becomes difficult to determine the effect of interest rate on call option value by simple financial intuition. Fortunately, thanks to the put-call symmetry relation for the prices of American vanilla call and put options (Kwok, 1998):

$$
C(S, t ; r, q, X)=P(X, t ; q, r, S)
$$

we can conclude that the vanilla call value $C(S, t ; r, q, X)$ is an increasing function of $r$. This is because the vanilla put price function $P(S, t ; r, q, X)$ is an increasing function of $q$ so that the modified put price function $P(X, t ; q, r, S)$ is an increasing function of $r$.

\subsection{MONOTONICITY PROPERTIES WITH RESPECT TO DIVIDEND YIELD AND INTEREST RATE}

For fixed strike path dependent options, the above intuitive arguments also hold. That is, the fixed strike call (put) value is monotonically decreasing (increasing) with respect to $q$ and the fixed strike put value is monotonically decreasing with respect to $r$. Unfortunately, we do not have the put-call symmetry relation for the fixed strike call and put. Hence, we cannot infer the monotonicity properties with respect to $r$ of the fixed strike call value, like those of the vanilla option counterparts. Indeed such monotonicity property does not hold. We shall show mathematically the rationale for the lack of such monotonicity and verify the phenomenon through numerical examples. For floating strike path dependent options, it is even more difficult to deduce the monotonicity properties from financial intuition. We shall show that the monotonicity properties with respect to $r$ exist for all floating strike path dependent options. However, only the floating strike call value has monotonicity property with respect to $q$. We first state the monotonicity properties for the values of European path dependent options, then generalize the results to their American counterparts.

\section{Price functions of European path dependent options}

The comparison principle in partial differential equation theory can be used to prove the monotonicity properties with respect to $q$ and $r$ for European path dependent options. The results for fixed strike and floating strike path dependent options are summarized in the following two lemmas.

\section{Lemma 6}

The price function of a European fixed strike path dependent call (put) option is monotonically decreasing (increasing) with respect to the dividend yield $q$ of the underlying asset. With an increase of the riskless interest rate $r$, the value of a European fixed strike put decreases. However, no monotonicity properties with respect to $r$ holds for a European fixed strike call. 


\section{Lemma 7}

The price function of a European floating strike path dependent call (put) is monotonically increasing (decreasing) with respect to the riskless interest rate $r$. For European floating strike options, only the call value has monotonicity (decreasing) property with respect to the dividend yield $q$.

The proof of Lemma 6 is presented in the Appendix. For Lemma 7, the proof of the ambiguity of monotonicity property with respect to $q$ of the floating strike put value is presented in the Appendix.

\section{Price functions of American path dependent options}

The above monotonicity properties with respect to $r$ and $q$ for the values of European path dependent options also hold for their American counterparts. To prove these claims, one may apply the penalty technique in partial differential equation theory (Jiang, 2002). Here, we would like to provide a simpler approach by proving the results under the discrete binomial tree model (Cox et al., 1979). Since the option values derived from the binomial tree models for American path dependent options have been shown to converge to their continuous counterparts (Jiang and Dai, 2004), we can infer the validity of the monotonicity properties for the option values of the continuous models from those of the discrete models.

Let us take the American Asian option as an example. The underlying asset price is assumed to follow the discrete binomial process. Let $\Delta t$ be the time step, $u$ and $d$ denote the proportional upward and downward jumps in the binomial process and $p$ denotes the probability of upward jump. The parameter values are given by (Cox et al., 1979)

$$
u=\frac{1}{d}=e^{\sigma \sqrt{\Delta t}}, p=\frac{e^{(r-q) \Delta t}-d}{u-d} .
$$

Let $V^{n}(S, A)$ denote the option value at the $n^{\text {th }}$ time step, with asset price $S$ and average asset value $A$. The discrete binomial pricing model for the American Asian option is given by (Jiang and Dai, 2004)

$$
\begin{aligned}
V^{n}(S, A)=\max ( & e^{-r \Delta t}\left[p V^{n+1}\left(S u, \frac{n A+S u}{n+1}\right)\right. \\
& \left.\left.+(1-p) V^{n+1}\left(S d, \frac{n A+S d}{n+1}\right)\right], \Lambda(S, A)\right)
\end{aligned}
$$

with terminal conditions:

$$
V^{N}(S, A)=\Lambda(S, A)
$$

where $\Lambda(S, A)$ is the payoff function and $N \Delta t=T$. Here, $N$ is the total number of time steps.

In the following exposition, we illustrate the proof on the monotonicity property with respect to $q$ for the American floating strike Asian call value. The proofs for other types of American path dependent options can be performed in a similar manner. We apply the transformation

$$
W^{n}(x)=\frac{V^{n}(S, A)}{S} \text { and } \quad x=\frac{S}{A}
$$

so that the binomial pricing scheme (3.3) can be reformulated as

$$
\begin{aligned}
W^{n}(x)=\max \left(e ^ { - r \Delta t } \left[p u W^{n+1}\left(\frac{n x d+1}{n+1}\right)\right.\right. & \\
& \left.\left.+(1-p) d W^{n+1}\left(\frac{n x u+1}{n+1}\right)\right], \phi(x)\right) \\
W^{N}(x)=\phi(x)= & (1-x)^{+} .
\end{aligned}
$$


To prove the monotonically decreasing property with respect to $q$ of the American floating strike Asian call, it suffices to show that

$$
W^{n}\left(x ; q_{1}\right) \geq W^{n}\left(x ; q_{2}\right) \text { for } \quad q_{1} \leq q_{2}
$$

The detailed proof of the above result is presented in the Appendix.

Exercise regions of American path dependent options

Once we have established the monotonicity properties with respect to $r$ and $q$ of the values of American path dependent options, we can deduce the corresponding monotonicity properties of the exercise regions of these options. These monotonicity properties are summarized in Proposition 8.

\section{Proposition 8}

We use $E_{f c}(r)\left[E_{f c}(q)\right]$ to denote the exercise region of the American floating strike call option with interest rate $r$ (dividend yield $q$ ), and similar notations for other types of American path dependent options. The exercise regions of various types of American path dependent options observe the following monotonicity properties with respect to the interest rate $r$ and dividend yield $q$.

1. Monotonicity properties with respect to $r$

Let $r_{1} \leq r_{2}$, we have

$$
\begin{aligned}
& E_{f c}\left(r_{2}\right) \subseteq E_{f c}\left(r_{1}\right) \\
& E_{f p}\left(r_{1}\right) \subseteq E_{f p}\left(r_{2}\right) \\
& E_{x p}\left(r_{1}\right) \subseteq E_{x p}\left(r_{2}\right) .
\end{aligned}
$$

There is no monotonicity property for the American fixed strike call options.

2. Monotonicity properties with respect to $q$

Let $q_{1} \leq q_{2}$, we have

$$
\begin{aligned}
& E_{x c}\left(q_{1}\right) \subseteq E_{x c}\left(q_{2}\right) \\
& E_{x p}\left(q_{2}\right) \subseteq E_{x p}\left(q_{1}\right) \\
& E_{f c}\left(q_{1}\right) \subseteq E_{f c}\left(q_{2}\right) .
\end{aligned}
$$

There is no monotonicity property for the American floating strike put options.

\section{Remarks}

1. We have performed sample calculations to demonstrate that the American fixed strike Asian put value is monotonically decreasing with respect to $r$ while its call option counterpart exhibits no such monotonicity (see Figure 1).

2. A simple intuitive argument can be presented to explain the loss of monotonicity property with respect to $q$ of the exercise regions. When $q=0$, it is known that the Russian option (perpetual American option with realized maximum asset value as its payoff) will never be exercised prematurely, thus implying that the perpetual floating strike lookback put will never be exercised prematurely too (Kwok, 1998). When $q>0, x^{*}(t)$ starts with a finite value and tends to $\max \left(\frac{q}{r}, 1\right)$ as time is approaching expiry. When $q=0, x^{*}(t)$ is infinite at infinite time to expiry but tends to 1 as time is approaching expiry. Therefore, 
at least for $q>r$, we argue that the corresponding $x^{*}(t)$ of the American lookback put will intersect $x^{*}(t)$ of the counterpart with $q=0$. Hence, the exercise regions of the American lookback put options do not have monotonicity property with respect to $q$.

\subsection{MONTONICITY PROPERTIES WITH RESPECT TO CALENDAR TIME AND MATURITY}

In this subsection, we analyze the monotonicity properties of the option values and exercise boundaries of American Asian and lookback options with respect to the interest rate, dividend yield and calendar time. The analysis of the monotonicity properties with respect to the volatility parameter is less interesting since option price functions are always increasing function of volatility while monotoncity with respect to interest rate and dividend yield may not always hold for all types of American options. For a good reference on related analysis on volatility effects, Jiang's paper (2002) presents the effective tool to analyze the monotonicity properties of American option price functions with respect to volatility.

A longer-lived American option must be worth at least its shorter-lived counterpart since the longerlived option has the additional right to exercise between the two expiration dates. Hence, the American option value is always monotonically increasing with respect to $T$ so that $\frac{\partial V}{\partial T} \geq 0$. Let $E(T)$ denote the exercise region of an American path dependent option with maturity date $T$. The monotonicity property of the option price function with respect to $T$ dictates that if $T_{1} \leq T_{2}$ then

$$
E\left(T_{1}\right) \supseteq E\left(T_{2}\right) \text { for both American Asian and lookback options. }
$$

For American lookback options, their values depend only on time to expiry $\tau=T-t$ so that

$$
\frac{\partial V}{\partial t}=-\frac{\partial V}{\partial T} \leq 0 .
$$

From the time-monotonicity property of the lookback option value, we can deduce the monotonicity of the exercise boundary with respect to the calendar time $t$. However, for American Asian options, their values depend on both $t$ and $T$ so that the monotonicity with respect to $t$ of the option value and exercise boundary do not hold [see the numerical examples demonstrated by Hansen and Jorgensen (2000)].

\section{Perpetual American path dependent options}

While most perpetual options do not exhibit dependence on the calendar time $t$, we observe that the values of American Asian options have dependence on $t$. Otherwise, other analytic properties of the perpetual American Asian options are similar to those of the lookback counterparts. For example, it is known that perpetual American lookback option values are finite when $q \neq 0$. We can deduce that perpetual American Asian option values are also finite since they are bounded by the values of the lookback counterparts. Furthermore, since the exercise regions of perpetual American lookback options are non-empty when $q \neq 0$, so those of the perpetual American Asian options are non-empty also. Interestingly, when $q=0$, the perpetual American floating strike Asian and lookback calls have the same value as that of the non-dividend paying underlying asset. We then deduce that it is never optimal to exercise these American call options prematurely, so their exercise regions are empty. To prove the claim, we use the put-call parity relation of the finite-lived European floating strike Asian options. Let $v_{A, f c}(S, J, t ; T)\left[v_{A, f p}(S, J, t ; T)\right]$ denote the price function of the finite-lived European floating strike call (put) option with maturity $T$. By establishing the following results

$$
S \geq V_{L, f c}(S, J, t ; \infty)
$$




$$
\begin{aligned}
& \geq V_{A, f c}(S, J, t ; \infty) \geq V_{A, f c}(S, J, t ; T) \geq v_{A, f c}(S, J, t ; T) \\
& =v_{A, f p}(S, J, t ; T)+\left[1-\frac{1}{r T}\right] S+\frac{S}{r T} e^{-r(T-t)}-\frac{t}{T} J e^{-r(T-t)} \\
& \geq\left[1-\frac{1}{r T}\right] S+\frac{S}{r T} e^{-r(T-t)}-\frac{t}{T} J e^{-r(T-t)} \rightarrow S \text { as } T \rightarrow \infty,
\end{aligned}
$$

we can deduce that

$$
V_{L, f c}(S, J, t ; \infty)=V_{A, f_{c}}(S, J, t ; \infty)=S
$$

\section{CONCLUSION}

We have developed a general framework of applying partial differential equation theory to analyze the monotonicity properties of the price functions and the optimal stopping regions of American path dependent options with either the Asian or lookback feature. From the ordering properties of the price functions of the American lookback options and their Asian option counterparts, we can deduce that the exercise region of an American lookback option is always contained in the exercise region of its Asian option counterpart. When the underlying asset pays a continuous dividend yield, we show that the exercise region exists for all times for any American Asian option. When the underlying asset is non-dividend paying, the exercise region also exists for all finite-lived American Asian option except that it is inconclusive to claim whether the exercise region of the American floating strike Asian call option exists for all times. Also, we have established some interesting monotonicity properties of the price functions and exercise regions of European and American path dependent options with respect to dividend yield, riskless interest rate and time. With the floating strike put as an exception, the price functions of all path dependent options are monotonic with respect to the dividend yield of the underlying asset. Except for the fixed strike call, the values of path dependent options also exhibit monotonicity properties with respect to the interest rate. We also find some interesting differences of the optimal exercise policies of the two classes of American path dependent option models. The exercise boundary of an American Asian option may not observe the monotonicity property with respect to the calendar time while the American lookback option counterpart always does so. The exercise region of some types of perpetual American path dependent options on a non-dividend paying asset may not exist. The perpetual American floating strike Asian and lookback calls have the same value as that of the non-dividend paying asset, so it is never optimal to exercise these call options prematurely.

The analyses presented above are based on the Black-Scholes pricing framework. Some of the techniques of analysis developed in this paper can be extended to pricing models under other stochastic price processes, like stochastic volatility model and jump-diffusion process. Since most of the ordering properties obtained in our work are model independent, we may deduce the pricing properties of the more complicated American Asian option models from those of the simpler American lookback option models.

\section{APPENDIX}

\section{Proof of Lemma 3}

To show that the stopping region lies on the left hand side of the stopping boundary for the American floating strike Asian call, it suffices to show that $W-(1-x)$ is a non-decreasing function of $x$. That is, it is necessary to establish

$$
\frac{\partial}{\partial x}[W-(1-x)]=\frac{\partial W}{\partial x}+1 \geq 0 .
$$


Since $\frac{\partial W}{\partial x}=\frac{\partial V}{\partial A}$, it is equivalent to prove

$$
\frac{\partial V}{\partial A} \geq-1
$$

It suffices to show

$$
\frac{V(S, A+\delta, \tau)-V(S, A, \tau)}{\delta} \geq-1 \quad \text { for any } \delta>0,
$$

or

$$
V(S, A+\delta, \tau)+\delta \geq V(S, A, \tau) \text { for any } \delta>0 .
$$

Let

$$
\bar{V}(S, A, \tau ; \delta)=V(S, A+\delta, \tau)+\delta,
$$

then $\bar{V}(S, A, \tau)$ satisfies the following variational inequalities:

$$
\begin{aligned}
& \frac{\partial \bar{V}}{\partial \tau}-\frac{S-A}{T-\tau} \frac{\partial \bar{V}}{\partial A}-\frac{\sigma^{2}}{2} S^{2} \frac{\partial^{2} \bar{V}}{\partial S^{2}}-(r-q) S \frac{\partial V}{\partial S}+r \bar{V} \geq r \delta-\frac{\delta}{T-\tau} \frac{\partial V}{\partial A}(S, A+\delta, \tau) \\
& \bar{V} \geq S-(A+\delta)+\delta=S-A \\
& {\left[\frac{\partial \bar{V}}{\partial \tau}-\frac{S-A}{T-\tau} \frac{\partial \bar{V}}{\partial A}-\frac{\sigma^{2}}{2} S^{2} \frac{\partial^{2} \bar{V}}{\partial S^{2}}-(r-q) \delta \frac{\partial \bar{V}}{\partial S}+r \bar{V}-r \delta+\frac{\delta}{T-\tau} \frac{\partial V}{\partial A}(S, A+\delta, \tau)\right]} \\
& {[\bar{V}-(S-A)]=0}
\end{aligned}
$$

with terminal condition:

$$
V(S, A, T)=(S-A-\delta)^{+}+\delta=\max (\delta, S-A) .
$$

Since $\frac{\partial V}{\partial A} \leq 0$ for the American floating strike call, we have

$$
r \delta-\frac{\delta}{T-\tau} \frac{\partial V}{\partial A}(S, A+\delta, \tau)>0 .
$$

By applying the comparison principle, we can deduce that

$$
\bar{V}(S, A, \tau) \geq V(S, A, \tau)
$$

which gives the desired result.

\section{Proof of Proposition 4}

We only consider the American floating strike Asian call. The proof for the put option counterpart is similar. In the exercise region, $W=1-x$. By substituting $W=1-x$ into the first variational inequality for $W$ [see Eq. (2.7)], we obtain

$$
\begin{aligned}
& \frac{\partial}{\partial \tau}(1-x)-\frac{\sigma^{2}}{2} x^{2} \frac{\partial^{2}}{\partial x^{2}}(1-x)+\left[(r-q) x-\frac{1-x}{T-\tau}\right] \frac{\partial}{\partial x}(1-x)+q(1-x) \\
= & (q-r) x+\frac{1-x}{T-\tau}+q(1-x) \geq 0,
\end{aligned}
$$

so that the optimal stopping region must be contained in the region

$$
\left\{(x, \tau):-r x+q+\frac{1-x}{T-\tau} \geq 0, \tau>0\right\} .
$$


This leads to

$$
x^{*}\left(0^{+}\right) \leq \frac{1+q T}{1+r T}
$$

Together with the requirement of non-negativity of the exercise payoff: $1-x^{*}\left(0^{+}\right) \geq 0$, we obtain

$$
x^{*}(0)^{+} \leq \min \left(\frac{1+q T}{1+r T}, 1\right)
$$

To show that the above inequality reduces to an equality, it suffices to show that $x^{*}\left(0^{+}\right) \geq \min \left(\frac{1+q T}{1+r T}, 1\right)$.

Assume the contrary, suppose there exists $\left(x, 0^{+}\right)$in the continuation region where $x<\min \left(\frac{1+q T}{1+r T}, 1\right)$. In the continuation region, $W\left(x, 0^{+}\right)=1-x$ and

$$
\left.\frac{\partial W}{\partial \tau}\right|_{\tau=0^{+}}=-\left[(q-r) x+\frac{1-x}{T-\tau}+q(1-x)\right]<0
$$

when $x<\min \left(\frac{1+q T}{1+r T}, 1\right)$. This leads to a contradiction with $\left.\frac{\partial W}{\partial \tau}\right|_{\tau=0^{+}}>0$ since $W(x, \tau) \geq(1-x)^{+}$and $W(x, 0)=(1-x)^{+}$. Hence, we deduce that

$$
x^{*}\left(0^{+}\right)=\min \left(\frac{1+q T}{1+r T}, 1\right) .
$$

\section{Proof of Proposition 5}

(a) We have shown that $A^{*}(S, \tau)>\frac{S+r T K}{1+r T}, \tau>0$ [see Eq. (2.11)]. Also, from the non-negativity of the exercise payoff, we have $A^{*}(S, \tau) \geq K, \tau>0$ so that $A^{*}\left(S, 0^{+}\right) \geq \max \left(K, \frac{S+r T K}{1+r T}\right)$. To show

$$
A^{*}\left(S, 0^{+}\right)=\max \left(K, \frac{S+r T K}{1+r T}\right)
$$

it suffices to show that $A^{*}\left(S, 0^{+}\right) \leq \max \left(K, \frac{S+r T K}{1+r T}\right)$. Assume the contrary, suppose $A^{*}\left(S, 0^{+}\right)>$ $\max \left(K, \frac{S+r T K}{1+r T}\right)$, then there exists a point $\left(S, A, 0^{+}\right)$in the continuation region where $A>\max \left(K, \frac{S+r T K}{1+r T}\right)$. At $\tau \rightarrow 0^{+}, V\left(S, A, 0^{+}\right)=A-K$, so we obtain

$$
\left.\frac{\partial V}{\partial \tau}\right|_{\tau=0^{+}}=-A+S-r T A+r T K<0 \text { since } A>\frac{S+r T K}{1+r T}
$$

This is a violation of the condition $\left.\frac{\partial V}{\partial \tau}\right|_{\tau=0^{+}}>0$ (condition for the option to remain alive in the continuation region). Combining all these arguments, we obtain the result in Eq. (i).

(b) From the non-negativity of the payoff of the fixed strike call, we have $A^{*}\left(0^{+}, \tau\right) \geq K, \tau>0$. Dai and Kwok $(2005)$ show that $M^{*}\left(0^{+}, \tau\right)=K$ for the fixed strike lookback call. From the nesting property of 
the exercise regions (see Lemma 2), we have $M^{*}\left(0^{+}, \tau\right) \geq A^{*}\left(0^{+}, \tau\right), \tau>0$. Combining all the results, we obtain

$$
A^{*}\left(0^{+}, \tau\right)=K \text {. }
$$

(c) When $K=0$, the payoff of the American fixed strike Asian call becomes linear homogeneous in $S$, like that of an American floating strike Asian option. Hence, we expect that $A^{*}(S, \tau ; 0)$ is also linear homogeneous in $S$ so that the ratio $\frac{A^{*}(S, \tau ; 0)}{S}$ is some function of $\tau$ [call it $\xi(\tau)$ ]. Furthermore, by observing that $A^{*}(S, \tau ; K)$ is linear homogeneous in $K$, we obtain

$$
\begin{aligned}
\lim _{S \rightarrow \infty} \frac{A^{*}(S, \tau ; K)}{S} & =\lim _{S \rightarrow \infty} \frac{A^{*}\left(\frac{S}{K}, \tau ; 1\right)}{\frac{S}{K}}=\lim _{K \rightarrow 0} \frac{A^{*}\left(\frac{S}{K}, \tau ; 1\right)}{\frac{S}{K}} \\
& =\lim _{K \rightarrow 0} \frac{A^{*}(S, \tau ; K)}{S}=\frac{A^{*}(S, \tau ; 0)}{S} .
\end{aligned}
$$

\section{Proof of Lemma 6}

Here, the European Asian option model is chosen as an illustration. Similar arguments of proof can be applied to European lookback option models. First, we show the monotonicity properties with respect to $q$. Let

$$
U(S, A, \tau)=V\left(S, A, \tau ; q_{1}\right)-V\left(S, A, \tau ; q_{2}\right), q_{1} \leq q_{2},
$$

where $V(S, A, \tau ; q)$ is the price function of a European fixed strike Asian option with dividend yield $q$. Since $V(S, A, \tau ; q)$ satisfies the following governing equation

$$
\frac{\partial V}{\partial \tau}-\frac{S-A}{T-\tau} \frac{\partial V}{\partial A}-\frac{\sigma^{2}}{2} S^{2} \frac{\partial^{2} V}{\partial S^{2}}-(r-q) S \frac{\partial V}{\partial S}+r V=0
$$

then $U(S, A, \tau)$ satisfies

$$
\frac{\partial U}{\partial \tau}-\frac{S-A}{T-\tau} \frac{\partial U}{\partial A}-\frac{\sigma^{2}}{2} S^{2} \frac{\partial^{2} U}{\partial S^{2}}-\left(r-q_{1}\right) S \frac{\partial U}{\partial S}+r U=\left(q_{2}-q_{1}\right) S \Delta\left(q_{2}\right)
$$

with initial condition: $U(S, A, 0)=0$. Here, $\Delta\left(q_{2}\right)$ denotes the option delta $\frac{\partial V}{\partial S}\left(S, A, \tau ; q_{2}\right)$. It is well known that $\Delta\left(q_{2}\right)$ is non-negative (non-positive) for a fixed strike call (put) so that

$$
\left\{\begin{array}{ll}
\left(q_{2}-q_{1}\right) S \Delta\left(q_{2}\right) \geq 0 & \text { for a fixed strike call } \\
\left(q_{2}-q_{1}\right) S \Delta\left(q_{2}\right) \leq 0 & \text { for a fixed strike put }
\end{array} .\right.
$$

Hence, we conclude from the comparison principle that

$$
\left\{\begin{array}{l}
U(S, A, \tau) \geq 0 \quad \text { for a fixed strike call } \\
U(S, A, \tau) \leq 0 \quad \text { for a fixed strike put }
\end{array} .\right.
$$

To show the monotonicity properties with respect to $r$, we consider

$$
\widehat{U}(S, A, \tau)=V\left(S, A, \tau ; r_{1}\right)-V\left(S, A, \tau ; r_{2}\right), r_{1} \leq r_{2},
$$


where $V(S, A, \tau ; r)$ is the price function of a European fixed strike option with riskless interest rate $r$. It is seen that $\widehat{U}(S, A, \tau)$ satisfies

$$
\begin{aligned}
& \frac{\partial \widehat{U}}{\partial \tau}-\frac{S-A}{T-\tau} \frac{\partial \widehat{U}}{\partial A}-\frac{\sigma^{2}}{2} S^{2} \frac{\partial^{2} \widehat{U}}{\partial S^{2}}-\left(r_{1}-q\right) S \frac{\partial \widehat{U}}{\partial S}+r_{1} \widehat{U} \\
= & \left(r_{2}-r_{1}\right)\left[-S \Delta\left(r_{2}\right)+V\left(S, A, \tau ; r_{2}\right)\right]
\end{aligned}
$$

with zero initial condition. Here, $\Delta\left(r_{2}\right)$ denotes the option delta $\frac{\partial V}{\partial S}\left(S, A, \tau ; r_{2}\right)$.

(i) For a fixed strike Asian put, we have $\Delta\left(r_{2}\right) \leq 0$ so that

$$
-S \Delta\left(r_{2}\right)+V\left(S, A, \tau ; r_{2}\right) \geq 0 .
$$

Hence, we can deduce that

$$
V\left(S, A, \tau ; r_{1}\right) \geq V\left(S, A, \tau ; r_{2}\right), \quad r_{1} \leq r_{2}
$$

(ii) For a fixed strike Asian call, however, the sign of $-S \Delta\left(r_{2}\right)+V\left(S, A, \tau ; r_{2}\right)$ is ambiguous. Therefore, no monotonicity property with respect to $r$ can be deduced for the European fixed strike Asian call value.

\section{Proof of Lemma 7}

The pricing model of a floating strike path dependent option can be reduced to an one-dimensional model by normalizing the price function by the asset price $S$ and using the similarity variable $x=A / S$ as the independent variable. To show the monotonicity property with respect to $q$ of the floating strike path dependent option value, we let

$$
\widetilde{U}(x, \tau)=W\left(x, \tau ; q_{1}\right)-W\left(x, \tau ; q_{2}\right), \quad q_{1} \leq q_{2},
$$

where $W(x, \tau ; q)$ is the price function of a floating strike Asian option with dividend yield $q$. We write $W^{\prime}\left(q_{2}\right)=\frac{\partial W}{\partial x}\left(x, \tau ; q_{2}\right)$. It is seen that $\widetilde{U}$ satisfies

$$
\begin{aligned}
& \frac{\partial \widetilde{U}}{\partial \tau}-\frac{\sigma^{2}}{2} x^{2} \frac{\partial^{2} \widetilde{U}}{\partial x^{2}}-\left[\left(q_{1}-r\right) x+\frac{1-x}{T-\tau}\right] \frac{\partial \widetilde{U}}{\partial x}+q_{1} \widetilde{U} \\
= & \left(q_{2}-q_{1}\right)\left[-x W^{\prime}\left(q_{2}\right)+W\left(x, \tau ; q_{2}\right)\right]
\end{aligned}
$$

with initial condition: $\widetilde{U}(x, 0)=0$. Recall that $\frac{\partial W}{\partial x} \leq 0$ for a floating strike Asian call while $\frac{\partial W}{\partial x} \geq 0$ for the put option counterpart. For a floating strike Asian call, we have $-x W^{\prime}\left(q_{2}\right)+W\left(x, \tau ; q_{2}\right) \geq 0$ so that the non-homogeneous term in the differential equation for $\widetilde{U}$ is always positive. By applying the comparison principle, we deduce that $\widetilde{U} \geq 0$ so that

$$
W\left(x, \tau ; q_{1}\right) \geq W\left(x, \tau ; q_{2}\right), \quad q_{1} \leq q_{2} .
$$

However, for a European floating strike Asian put, the sign of $-x W^{\prime}\left(q_{2}\right)+W\left(x, \tau ; q_{2}\right)$ is ambiguous so that no monotonicity property with respect to $q$ can be deduced for the floating strike Asian put.

Proof of inequality (3.5) 
Suppose we let

$$
p_{i}=\frac{e^{\left(r-q_{i}\right) \Delta t}-d}{u-d}, \quad i=1,2
$$

we can rewrite $W^{n}\left(x ; q_{1}\right)$ as follows:

$$
\begin{aligned}
& W^{n}\left(x ; q_{1}\right) \\
= & \max \left\{\frac{1}{\rho}\left[p_{1} u W^{n+1}\left(\frac{n x d+1}{n+1} ; q_{1}\right)+\left(1-p_{1}\right) d W^{n+1}\left(\frac{n x u+1}{n+1} ; q_{1}\right)\right], \phi(x)\right\} \\
= & \max \left\{\frac{e^{-q_{1} \Delta t}}{u-d}\left[u W^{n+1}\left(\frac{n x d+1}{n+1} ; q_{1}\right)-d W^{n+1}\left(\frac{n x u+1}{n+1} ; q_{1}\right)\right]\right. \\
& \left.+\frac{e^{-r \Delta t}}{u-d}\left[W^{n+1}\left(\frac{n x u+1}{n+1} ; q_{1}\right)-W^{n+1}\left(\frac{n x d+1}{n+1} ; q_{1}\right)\right], \phi(x)\right\} .
\end{aligned}
$$

By observing that $W^{n+1}\left(x_{1}\right) \geq W^{n+1}\left(x_{2}\right)$ if $x_{1} \leq x_{2}$, we can establish

$$
u W^{n+1}\left(\frac{n x d+1}{n+1} ; q_{1}\right)-d W^{n+1}\left(\frac{n x u+1}{n+1} ; q_{1}\right) \geq 0 .
$$

We apply the induction argument. Suppose inequality (3.5) is valid for $n+1$, then by the assumption of induction, we obtain

$$
\begin{aligned}
& W^{n}\left(x ; q_{1}\right) \\
\geq & \max \left\{\frac{e^{-q_{2} \Delta t}}{u-d}\left[u W^{n+1}\left(\frac{n x d+1}{n+1} ; q_{1}\right)-d W^{n+1}\left(\frac{n x u+1}{n+1} ; q_{1}\right)\right]\right. \\
& \left.+\frac{e^{-r \Delta t}}{u-d}\left[W^{n+1}\left(\frac{n x u+1}{n+1} ; q_{1}\right)-W^{n+1}\left(\frac{n x d+1}{n+1} ; q_{1}\right)\right], \phi(x)\right\} \\
= & \max \left\{\frac{1}{\rho}\left[p_{2} u W^{n+1}\left(\frac{n x d+1}{n+1} ; q_{1}\right)+\left(1-p_{2}\right) d W^{n+1}\left(\frac{n x u+1}{n+1} ; q_{1}\right)\right], \phi(x)\right\} \\
\geq & \max \left\{\frac{1}{\rho}\left[p_{2} u W^{n+1}\left(\frac{n x d+1}{n+1} ; q_{2}\right)+\left(1-p_{2}\right) d W^{n+1}\left(\frac{n x u+1}{n+1} ; q_{2}\right)\right], \phi(x)\right\} \\
= & W^{n}\left(x ; q_{2}\right),
\end{aligned}
$$

where the last inequality is due to the assumption of induction, $p_{2}>0$ and $\left(1-p_{2}\right)>0$. Therefore, we see that $W^{n}(x)$ decreases as $q$ increases for the American floating strike Asian call. Following similar argument, we can show the monotonicity property with respect to $r$ for the American fixed strike Asian option values.

\section{Remark}

For the American floating strike Asian put option, we have

$$
W^{n+1}\left(x_{1}\right) \leq W^{n+1}\left(x_{2}\right) \quad \text { if } \quad x_{1} \leq x_{2} .
$$

As a result, there is ambiguity about the sign of

$$
u W^{n+1}\left(\frac{n x d+1}{n+1}\right)-d W^{n+1}\left(\frac{n x u+1}{n+1}\right) .
$$

Hence, we cannot deduce the monotonicity property with respect to $q$ for the value of the American floating strike Asian put option. 


\section{REFERENCES}

BEN-AMEUR, H., M. BRETON and P. L'ECUYER (2002): A dynamic programming procedure for pricing American-style Asian options. Management Science 48(5), 625-643.

CHIARELLA, C. and A. ZIOGAS (2004): McKean's method applied to American call options on jumpdiffusion processes. Working paper of University of Technology, Sydney.

COX, J.C., S. ROSS and M. RUBINSTEIN (1979): Option pricing: a simplified approach. Journal of Financial Economics 7, 229-264.

DAI, M. (2001): Numerical analysis on binomial tree methods for American lookback options. Numerical Mathematics: Journal of Chinese Universities 10(2), 170-181.

DAI, M. and Y.K. KWOK (2005): American options with lookback payoff. Working paper [can be downloaded at http://www.math.ust.hk/ maykwok].

DAI, M., Y.K. KWOK and L.X. WU (2004): Optimal shouting policies of options with strike reset right. Mathematical Finance 14(3), 383-401.

GUKHAL, C.R. (2001): Analytical valuation of American options on jump-diffusion processes. Mathematical Finance 11, 97-115.

d'HALLUIN Y., P.A. FORSYTH, and G. LABAHN (2003): A semi-Lagrangian approach for American Asian options under jump diffusion. Working paper of University of Waterloo.

HANSEN, A.T. and P.L. Jorgensen (2000): Analytical valuation of American-style Asian options. Management Science 46(8):1116-1136.

JAILLET, P., D. LAMBERTON and B. LAPEYRE (1990): Variational inequalities and the pricing of American options. Acta Applicandae Mathematicae 21, 263-289.

JIANG, L.S. (2002): Analysis of pricing American options on maximum (minimum) of two risky assets. Interfaces and Free Boundaries 4, 27-46.

JIANG, L.S. and M. Dai (2004): Convergence of binomial tree method for European/American path-dependent options. SIAM Journal of Numerical Analysis 42(3), 1094-1109.

KWOK, Y.K. (1998): Mathematical models of financial derivatives. Springer, Singapore.

LAI, T.L. and T.W. Lim (2004): Exercise regions and efficient valuation of American lookback options. Mathematical Finance 14(2), 249-269.

MARCOZZI, M.D. (2003): On the valuation of Asian options by variational methods. SIAM Journal of Scientific Computing 24(4), 1124-1140. 
PAROTT, K. and N. CLARKE (1998): A parallel solution of early exercise Asian options with stochastic volatility. Proceedings of the 11th Domain Decomposition Conference, Greenwich.

PHAM, H. (1997): Optimal stopping, free boundary and American option in a jump-diffusion model. Applied Mathematics and Optimization 35, 145-164.

WU, L.X., Y.K. KWOK and H. YU (1999): Asian options with the American early exercise feature. International Journal of Theoretical and Applied Finance 2, 101-111.

WU, R. and M.C. FU (2003): Optimal exercise policies and simulation-based valuation for American-Asian options. Operations Research 51(1), 52-66.

YU, H., Y.K. KWOK and L. WU (2001): Early exercise policies of American floating and fixed strike lookback options. Nonlinear Analysis 47, 4592-4602. 

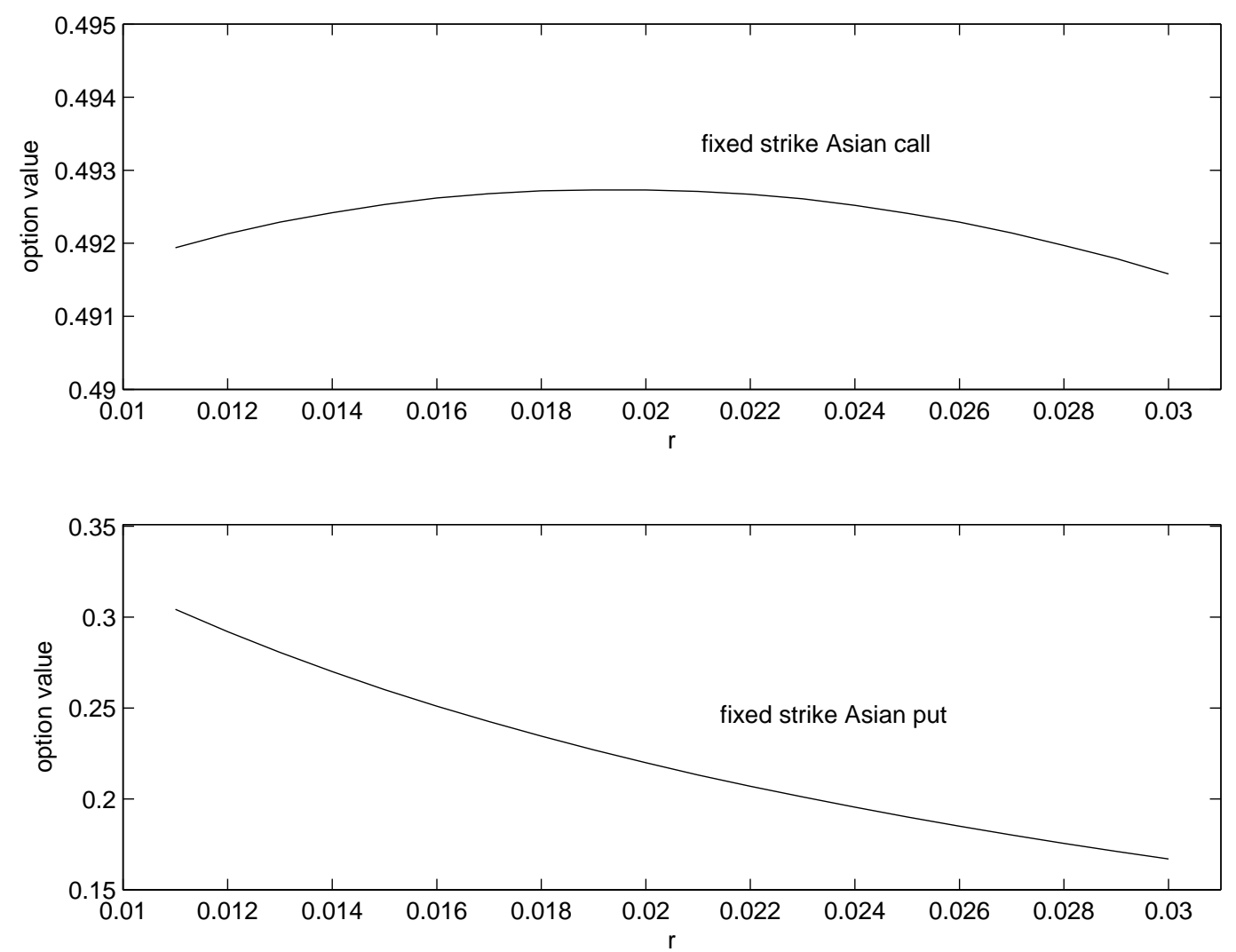

FIGURE 1. The American fixed strike Asian put value is monotonically decreasing with respect to the riskless interest rate $r$ while its call option counterpart exhibits no such monotonicity property. The parameter values of the option models are: $t=0, T=20, S=A=K=1, \sigma=0.4$ and $q=0$. 